\title{
Spontaneous distillation of silica-bearing solution in closed system with rough walls
}

\author{
Victor Alekseyev ${ }^{1, *}$, Victor Balashov ${ }^{2}$, Ludmila Medvedeva ${ }^{1}$, and Anton Opolchentsev ${ }^{3}$ \\ ${ }^{1}$ Vernadsky Institute of Geochemistry and Analytical Chemistry, Russian Academy of Sciences, \\ Moscow, 119991 Russia \\ ${ }^{2}$ Earth and Environmental Systems Institute, Pennsylvania State University, PA 16802, United States \\ ${ }^{3}$ Shubnikov Institute of Crystallography, Russian Academy of Sciences, Moscow, 119333 Russia
}

\begin{abstract}
The $300^{\circ} \mathrm{C}$ experiments were conducted in hermetic platinum ampoules with aqueous solution saturated by $\mathrm{SiO}_{2}$ with respect to quartz. The solute $\mathrm{SiO}_{2}$ concentration did not change with time in ampoules with the smooth walls, however in the ampoules with the induced roughness on their walls it decreased exponentially with time and amorphous silica precipitated on the ampoule walls contacted with the water vapor. The experimental results were explained by the distillation initiated through preferential water evaporation in the thin layer $(<100 \mathrm{~nm})$ at the meniscus edge and strengthened by the surface wall roughness because of the evaporation surface area increase at the wall wetting in the impregnation regime. This process was accompanied by the progressive disturbance of the equilibrium distribution of the dissolved silica between liquid water and vapor. The similar mechanism might exist in the systems of the different composition. It may initiate the continuous renewal of the water dissolving ability, which can lead at natural conditions to the migration of the voids partially filled by the solution.
\end{abstract}

\section{Introduction}

Water-rock interaction is studied using the quantitative data of thermodynamics, kinetics and dynamics based on the experimental and theoretical research of macrophenomena where the surface energy contribution is negligible. However in the last time the geochemists became more attentive to the nanoparticles and nanofluids $(<100 \mathrm{~nm})$, which are ubiquitous in nature but they have properties which are different from their macro-analogs [1]. One of the nanofluid example is the meniscus edge of liquid wetting the solid wall [2]. The thin $(<100 \mathrm{~nm})$ slightly overheated liquid layer is evaporated much faster than the same liquid located far from the wall. In the closed equilibrium system this property must be compensated by the preferable condensation over the bulk liquid surface, and it can lead to the distillation of aqueous solution. Namely, we proposed such mechanism for the explanation of the irreversible $\mathrm{SiO}_{2}$ transfer from the liquid water into the $\mathrm{SiO}_{2}$ solid phases situated in vapor [3-4]. In the last work, the acceleration of the $\mathrm{SiO}_{2}$ transfer was found for the case of the rough textured wall. In the present work, this research

\footnotetext{
*Corresponding author: alekseyev-v@geokhi.ru
} 
was continued to measure the process rate and to understand the mechanistic effect of the roughness on the $\mathrm{SiO}_{2}$ transfer.

\section{Methods}

The experiments were conducted in 2 stages. At the beginning, the crushed quartz was heated with distilled water at $300^{\circ} \mathrm{C}$ in autoclave for 3 days. Over this time, the water was saturated by silica with respect to quartz $(10.3 \mathrm{mmol} / \mathrm{kg})$. After the autoclave quenching this solution was poured by 0.5 g portions into 9 platinum ampoules each $8 \mathrm{~mm}$ in diameter. The ampoules were hermetically welded and heated at $300^{\circ} \mathrm{C}$ in the vertical autoclaves placed within the upper or lower part of an electric furnace where the temperature decreased $(-0.08 \% \mathrm{~cm})$ or increased $(0.15 \% \mathrm{~cm})$ upward [3]. Upon the specified heating time there were conducted the autoclave quenching and the ampoule opening, filtration, dilution and analysis of the aqueous solutions for the silica content (ICP AES). The first 2 experimental series were carried out with new ampoules in the upper and lower part of an electric furnace. The next series - with the same ampoules but their inner walls were made rough textured through polishing by quartz powders of the different grain size $(\mu \mathrm{m})$ : 63-100 (amp. 1, 2, 3); 20-50 (amp. 4, 5, 6); 10-20 (amp. 7, 8, 9). After the experimental series, the ampoules were cut along and their inner walls were studied using scanning electron microscope (SEM), energy dispersive analysis (EDA), contact profilometer (CP) and atom force microscope (AFM).

\section{Results}

In the new ampoules the concentration of silica dissolved in liquid water $(m)$ did not change after 4 days either at upper or lower part of an electric furnace. In the same ampoules but with the rough textured walls the concentration $m$ decreased in time (fig. 1). It is explained by the distillation, manifested itself through the process of continuous replacement of the water, contained $\mathrm{SiO}_{2}$, on the condensate [4]:

$$
m=\left(m_{0}-m_{v}\right) \exp \left(-\frac{J S t}{M}\right)+m_{v},
$$

where $m_{0}$ - the initial silica concentration in the solution, $m_{v}$ - the silica concentration in the water vapor (the condensate), $J$ - the specific rate of the preferential evaporation, $S-$ the surface area of the preferential evaporation, $t-$ time, $M-$ the water mass.

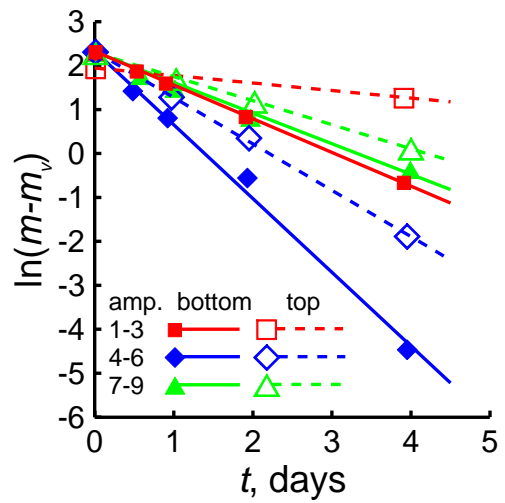

Fig. 1. Approximation of the experimental data (symbols) by the equation (1) (lines).

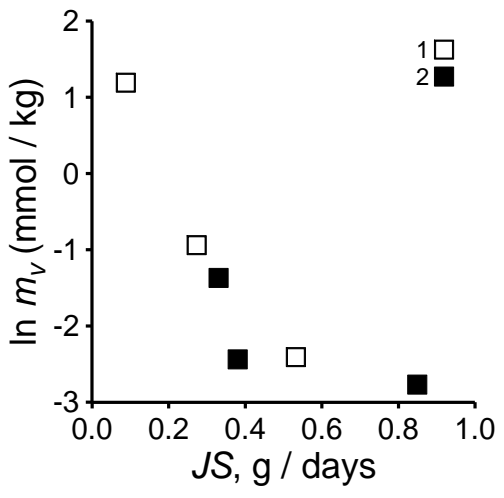

Fig. 2. Dependence $\ln m_{v}-J S$ for the upper (1) and lower (2) ampoules. 


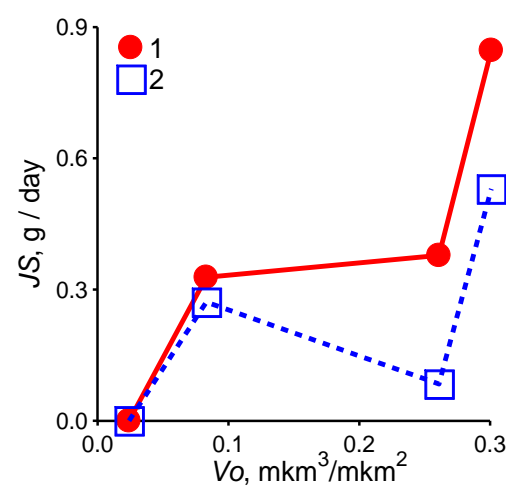

Fig. 3. Dependence of the evaporation rate $(J S)$ on the oil retaining volume (Vo) for the lower (1) and upper (2) ampoules.

Accordingly to Eq.(1), for large time $m=m_{v}$. Because of it the values of $m_{v}$ were taken from the long time experiments $(t>>4$ сут) with minimal values of $m$. The values of $J S$ were calculated from the slope of lines which approximated the experimental data in the coordinates $\ln \left(m-m_{v}\right)-t$ by the least square method (fig. 1). With the increase of $J S$ to 0.85 $\mathrm{g} /$ day the $m_{v}$ decreased to $0.06 \mathrm{mmol} / \mathrm{kg}$ (fig. 2). The last number is close to the quartz solubility in the water vapor [5].

The profilometer measurements of the rough textured ampoules 1-3, 4-6 and 7-9 indicated the next values of $R a(\mu \mathrm{m}): 3.0,2.3$ и $1.2(0.5 \mu \mathrm{m}$ for the new ampoules). The only roughness parameters, which changed regularly with the $J S$, were the $R k$-parameters, describing the cumulative curve of the profile ordinates (the Abbott curve) in the region of concavities. For example, oil-retaining volume $V o$, which characterizes the volume of the deepest concave hollows (valleys). For the lower ampoules, $J S$ increases regularly with the $V o$ increase, although for the upper ampoules the dependence is more complicated and the values of $J S$ are less (fig. 3). It can be the consequence of the partial water condensation on the walls as result of the upward temperature decrease.

The decrease of the $m$ was accompanied by the amorphous silica precipitation on the walls in the upper part of the ampoules (fig. 4a). The precipitate was in the form of spherical aggregates (fig. 4b) building the crust with the sharp boundaries. It was situated above the solution level (marked by the cross dark rim in the middle of the ampoule in Fig. 4a). It shows that the solution rose up the rough textured wall in the process of the evaporation.

The solution rise is explained by the wall wetting in the impregnation regime, when the solution rises over the hollows of the rough textured surface under the action of the capillary forces. At this wetting, the projections of the wall texture are left dry [6].
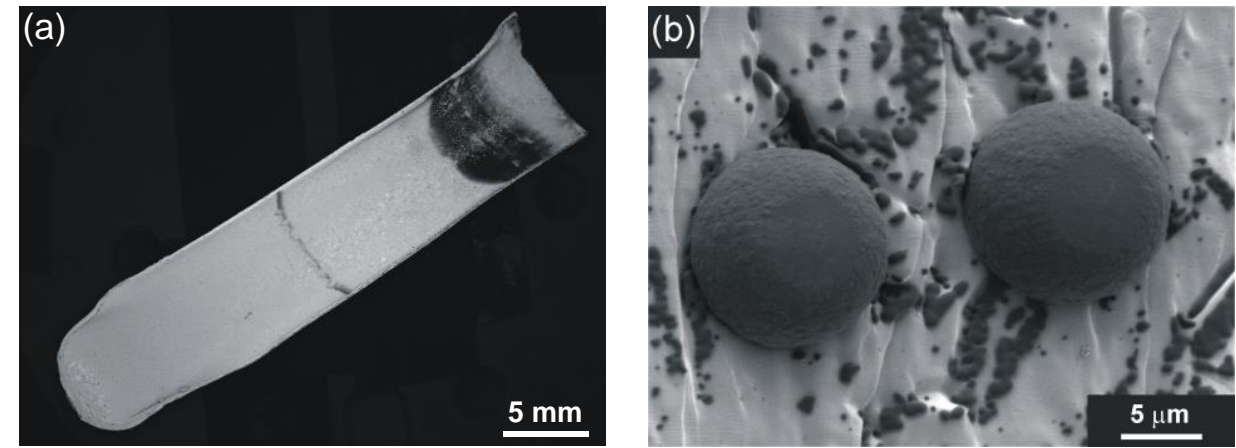

Fig. 4. The SEM-photo of the inner ampoule wall after experiment. (a) - the general outlook, (b) - the multiplication of the upper part. Light - platinum, dark - amorphous silica. 


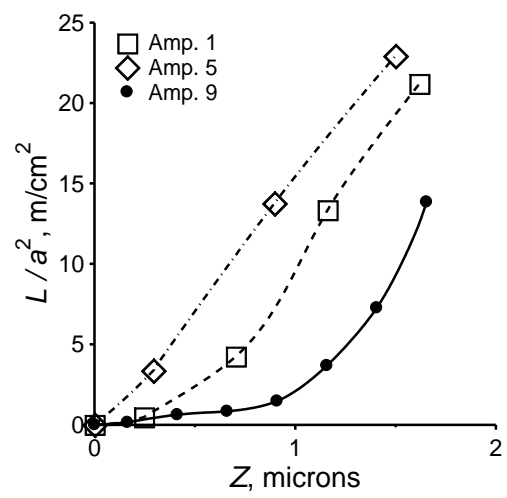

Fig. 5. The specific length of contact line of the solution with the surface projections at the different solution table measured from the deepest hollow.

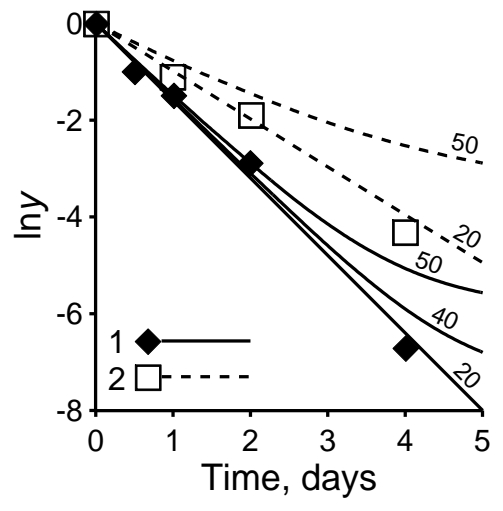

Fig. 6. The saturation degree $(y=(m$ $\left.\left.m_{v}\right) /\left(m_{e q}-m_{v}\right)\right)$ of the solution in the experiment (symbols) and in the model (lines) at the different solution film thickness $(\mu \mathrm{m}) . J S=0.85$ (1) and 0.53 (2) g/day.

This regime is supported by the hydrophilicity of the surface: at $300^{\circ} \mathrm{C}$ the equilibrium contact angle of the water on the smooth platinum $(\theta)$ is close to $0^{\circ}$ [7]. On the basis of the AFM data, there were build the sections of the rough textured surface parallel to the middle plane. For each section it was calculated the specific length of the contact line $\left(L / a^{2}\right)$ dividing the surface projections and valleys (it is assumed that the valleys can be filled by solution). As it follows from the fig. 3, the main interest has the sections of the deepest valleys (fig. 5). In this region, the $L / a^{2}$ increase took place for the same ampoules sequence (9-1-5) as the $J S$ increase within lower part of an electric furnace (fig. 1). For the mean $L / a^{2}=10 \mathrm{~m} / \mathrm{cm}^{2}$ the $L$ experimental value is on the 3 orders of magnitude greater than for the smooth wall, that explains the accelerated influence of the roughness on the distillation rate.

\section{Discussion}

Additionally to evaporation/condensation, at least two processes can affect the $m$ value: 1 ) the reverse $\mathrm{SiO}_{2}$ diffusion in the solution film and 2) the tendency of the dissolved silica to the equilibrium between liquid water and vapor.

The simplified reactive transport model of the distillation in quasi-stationary approximation was developed. It is based on the two mass transfer equations. Let us assume that the stationary water retained by the rough textured ampoule walls can be integrated into the cylindrical water film of height $L_{2}$ and thickness $h_{0}$. The aqueous fluid is unsaturated with respect to solid silica within the lower region of this film where the vertical coordinate $(z) 0 \leq z \leq L_{1}$ and the dissolved $\mathrm{SiO}_{2}$ transfer is described as

$$
J_{S i O_{2}}=\frac{J S u}{\pi d h_{0}}-D \rho_{w} \frac{d u}{d z}=C
$$

where $J_{\mathrm{SiO}_{2}}$ is the aqueous silica flux, $u=m-m_{v}, d$ is the ampoule diameter, $D-$ the aqueous silica diffusion coefficient, and $\rho_{w}-$ the water density. The fluid is supersaturated by silica within the upper film region $L_{1} \leq z \leq L_{2}$ and the $\mathrm{SiO}_{2}$ transfer with the solid silica precipitation is described by the equation 


$$
-\frac{d J_{S_{i O}}}{d z}=k h_{0}^{-1}\left(\frac{m}{m_{e q}}-1\right)
$$

where $\mathrm{k}$ is the kinetic constant. $C$ and $L_{l}$ are the functions of the time.

The coincidence of the experimental and model data was observed only at the model film thicknesses less than $20 \mu \mathrm{m}$ (fig. 6). At these thicknesses, the model corresponds to eq. (1). This is in an agreement with figs. 3, 5 and it evidences the lack of the reverse diffusion effect on $m$. Accordingly to the model the lower boundary $\left(L_{l}\right)$ of the supersaturation region moves upward in time that agrees with $\mathrm{SiO}_{2}$ precipitation in the form of enough wide rim (fig. 4a).

The equilibrium ratio $m / m_{v}=214$ [5], in the experiments it decreased and at large time it approached to 1, i.e. the system evolved away off equilibrium. It was promoted by the dissolution of amorphous silica in vapor, which supported $m_{v}$ at the constant level. However, the different $m_{v}$ values at the different $J S$ values (fig. 2) are still unexplained.

\section{Conclusion}

The distillation mechanism, described in the presented work, can be active in the geochemical systems of different compositions. The favorable factors for the distillation appearance are the presence of two water phases (liquid and vapor), the often occurrence of rough textured surfaces, hydrophilicity of the rock and mineral surface [8], the $\theta$ decrease with temperature increase [9]. The thermal gradient is the factor of the second order.

The special feature of this process is in a constant renewal of the dissolving ability of liquid water situated at the geo-cavity bottom, and the precipitation of secondary minerals at the upper part of the cavity. In the result, the cavity with a moderate amount of water has the ability to move down and to recrystallize during the way large rock volumes. This mechanism can explain the often-observable asymmetry of the natural crystallization cavities [10], which manifests itself in the predominance of large mineral crystals at the upper parts of cavities.

\section{References}

1. Y. Wang, Chem. Geol. 378-379, 1-23 (2014)

2. J.L. Plawsky, M. Ojha, A. Chatterjee, P.C. Wayner, Chem. Engin. Commun. 196, 658696 (2008)

3. V.A. Alekseyev, L.S. Medvedeva, Geochem. Int. 56, 136-147 (2018)

4. V.A. Alekseyev, L.S. Medvedeva, V.N. Balashov, A.A. Burmistrov, I.N. Gromiak, Geochem. Int. 56, 617-627 (2018)

5. A.V. Plyasunov, Geochim. Cosmochim. Acta 77, 215-231 (2012)

6. J. Bico, C. Tordeux, D. Quéré, Europhys. Lett. 55, 214-220 (2001)

7. B. Shi, S. Sinha, V.K. Dhir, American Society of Mechanical Engineers, Heat Transfer Division, (Publication) HTD, 376 HTD (1), 93-97 (2005)

8. A. Mazurek, S.J. Pogorzelski, K. Boniewicz-Szmyt, Oceanologia 51, 377-403 (2009)

9. A.W. Adamson, A.P. Gast, Physical chemistry of surfaces. 6th ed. (John Wiley \& Sons, New York, 1997)

10. A.M. Ashabov, Crystal genesis and evolution of “crystal-environment" system. (Nauka, Saint-Petersburg, 1993 in Russian) 